\title{
NUMERICAL SIMULATION OF THE OPEN-POOL REACTOR COOLANT SYSTEM USING A MULTI-DOMAIN APPROACH
}

\author{
Santiago F. Corzo ${ }^{1}$, Dario Godino ${ }^{1}$, Antonella Costa ${ }^{2,3}$, \\ Patricia A.L. Reis ${ }^{2,3}$, Claubia Pereira ${ }^{2,3}$ and Damian E. Ramajo ${ }^{1}$ \\ ${ }^{1}$ CIMEC Centro de Investigacion de Metodos Computacionales \\ UNL, CONICET, FICH \\ Col. Ruta 168 s/n, Predio Conicet "Dr Alberto Cassano" \\ 3000 Santa Fe, Argentina \\ ${ }^{2}$ Departamento de Engenharia Nuclear \\ Universidade Federal de Minas Gerais (UFMG) \\ Av. Antônio Carlos No. 6627 \\ Campus Pampulha, CEP 31270-901 \\ Belo Horizonte, MG, Brazil \\ ${ }^{3}$ Instituto Nacional de Ciências e Tecnologia \\ de Reatores Nucleares Inovadores/CNPq, Brazil
}

The computational simulation of large-scale reactors is currently limited by the high computational cost. The system codes allow addressing these problems, although with the well-known loss of local information. The use of coupling domains to reduce the problems looks like a proper alternative to settle this issue. In the present paper, a multi-domain coupling 3-dimensional/0-dimensional method to solve the thermal hydraulics of the TRIGA Mark I IPR-R1 reactor was implemented into a Finite Volume suite. Despite of the broadly literature about coupling methods, even in the nuclear engineering community, most of them manage with different codes in a fully explicit way. In the other hand, the benefit of solve different domain approaches inside the same software is in the use of monolithic algorithms. The proposed method consists on using 3-dimensional full CFD to simulate the reactor pool and 0-dimensional modelling for the external cooling loop. This is made by implementing a set of ad-hoc dynamics boundary conditions to model the momentum and energy balances along the pipeline. This strategy was used to perform long-time steady state simulations of the reactor at the design power of $100 \mathrm{~kW}$ as well as for the repowering up to $265 \mathrm{~kW}$. The results demonstrated that the core is efficiently cooled at the higher power without need to increase the coolant mass flow rate of the external system. Moreover, two accidental events were simulated: the first case was the Station Black Out at full power of $265 \mathrm{~kW}$. The results indicated that the loss 
of the external heat sink led to a slow pool heating, but the core remains being cooled by the natural circulation in the pool. In fact, the mass flow rate through the core is only reduced in $15 \%$ by the loss of the external loop circulation. Finally, a large-Loss of Coolant Accident for the operational power of $100 \mathrm{~kW}$ and keeping the pump running is performed. In this case, the pool is quickly empty if safety systems do not take action and the core is uncovered after $450 \mathrm{~s}$ completely losing the core cooling capacity.

\section{INTRODUCTION}

The thermal hydraulic analysis is an essential aspect in the design and assessment of power and research reactors to ensure safety operation conditions under steady-state and transient events. Despite research reactors are simpler than power plants, their behaviour under safety accident events should be investigated. These studies are commonly carried on using system codes. The use of three-dimensional Computational Fluid Dynamics (CFD) for assessing large installations is currently a challenge due to the computational cost limitations.

This paper studies the response of the TRIGA MARK I IPR-R1 research reactor under two accidental scenarios: A large Loss of Coolant Accident (LOCA), and a Station Black Out (SBO). A scheme of the reactor is shown in Figure 1. The hot coolant is extracted from the lower part of the pool (below the core) by the main pump. The water enters into a shell and tube heat exchanger, where the heat is transferred to a secondary loop and delivered to the atmosphere through of a cooling tower. Finally, the cooled water returns to the reactor pool. The Figure 1 also display views of the pool reactor and the core. The core contains the fuel elements, the aluminium cladding, the stainless-steel cladding, and some graphite elements. The core is surrounded by an annular graphite reflector. The rod elements are fixed by two supporting plates at the top and the bottom sides, which have orifices through which the coolant flows upward.

It is of crucial significance to predict the behaviour of the devices associated to the whole installation during long-time plant accidents such as Large-LOCA, LOFC, and Station Black-Out (SBO), among others. Such studies are currently impracticable using full 3-dimensional (3D) models. The use of $0 \mathrm{D}$ numerical models with large number of empirical correlations is widely accepted in the nuclear engineering. For more than 40 years the researches have performed 0-dimensional (0D) calculus to analyse start-up procedures and cost-down flow, among others, in real nuclear plants through thermal balances [1][2]. The nuclear engineering community has made much effort to development 0-dimensional/1-dimensional (0D/1D) system codes like RELAP [3], ATHLET [4], and TRACE, which are based on the domain reduction techniques combined with empirical correlations to model complex 
multiphase flows. These codes are widely employed for designing and assessing of nuclear power plants [5][6][7][8]. Of course, the great advantage of the analytical and semi-analytical approaches is in the low computational cost. However, the $0 \mathrm{D} / 1 \mathrm{D}$ codes do not provide detailed information regarding the flow inside complex devices.

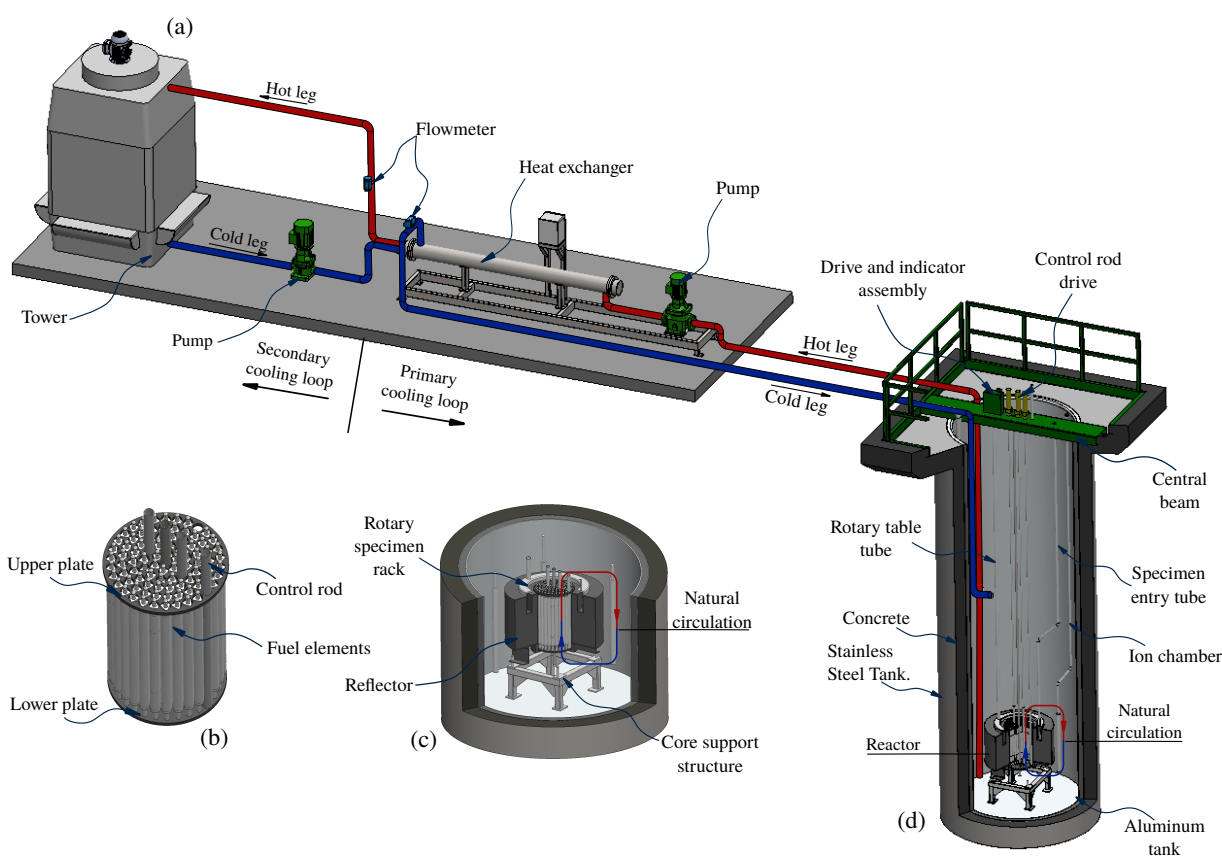

Figure 1: Schematic representation of the TRIGA MARK I IPR-I: (a) Cooling loop - (b) View of the fuel elements - (c) View of the reactor core - (d) View of the reactor pool [9]

Based on the aspects above discussed, the future use of CFD in nuclear engineering as well as in any other industrial application, should be focused on the development of multi-dimensional tools conceived to combine full 3D models for the main devices with $0 \mathrm{D}$ and $1 \mathrm{D}$ models for the surrounding components.

There are many publications addressing the coupling between system codes and 3D CFD. Only a few apply semi-implicit or implicit techniques, while the most of them are based on the use of explicit techniques leading to weak coupling. In these, the system code and the 3D CFD code run independently, exchanging the necessary data in an iterative way. Bandini et al. [10] have presented a detailed compilation of the publications concerning to the code-coupling tools to simulate new-generation reactors. The author has pointed out special emphasis in the validation of these tools respect to experimental facilities.

One of the first attends was done by Martin et al. [11], who coupled RELAP-5 with other codes using an explicit method by transferring data 
through a Parallel Virtual Machine (PVM) protocol. In the same way, Aumiller et al.[12] explicitly coupled RELAP5-3D with a CFD code showing good results. However, numerical instabilities were observed and reported by the authors. This were attributed to the explicit coupling algorithm. A much more recent developments using explicit methods were done to couple RELAP and CFX [13], ATHLET with OpenFOAM [14], and Star-CCM with TRACE [15]. In all cases the system codes take a master role, sending some information (mass flow, pressure, temperature) to the CFD codes. This mass-flow/pressure coupling is quite simple, but this could present stability problems for large time steps or fast flow variations [16].

In order to eliminate the numerical instabilities associated with the explicit methodology, Weaver et al. [17] developed a generic semi-implicit coupling technique. The master/slave communication among codes was also established, but the method performs the data communication among both codes. In contrast to explicit coupling, where the pressure in the coupled volumes is kept constant and upgraded at the end of the time step, in this method both the velocity and pressure are upgraded in each communication step. This coupling methodology has shown good performance in several flow conditions [18], and recently a complete description of the strength and weakness of the algorithm has been published by Aumiller et al. [19].

More recent coupling methods, such as the Dynamic-Implicit Additional Source (DIAS), have demonstrated to be a robust way to coupling system codes with sub-channel and CFD codes allowing to carry out multi-scale thermal-hydraulics simulations based on the domain-overlapping approach [20].

The use of 0D and 1D approaches through native CFD-code tools such as the External User Functions (EUF) has been also used to couple user codes inside of the framework of the CFD codes. The last has turned out more robust than weak coupling because the external 0D/1D code takes a slave role providing information that is updated inside the pressure-velocity coupling solver, leaving to a semi-implicit method. The authors have implemented EUF in the ANSYS-CFX framework to simulate the thermal-hydraulics of the overall pressure Vessel of the Pressurized Heavy Water Reactor Atucha II. They used a large 3D domain to simulate the core plenums and the downcomer coupled it with the 451 coolant channels housed inside the moderator tank. First they represented the coolant channels through a $0 \mathrm{D}$ ad hoc code [21]. Then, the code was improved by using a 1D single phase approach [22]. Finally, a two-phase model including pressure drop and sub-cooled boiling was performed [23].

In the current paper a 0D model is coded inside of Dynamic Boundary Conditions (DBC) in the OpenFOAM suite. These DBC impose timedependent Dirichlet values for the velocity and temperature at the boundaries of 3D CFD domains. The main advantage of the proposed tool is in the semi-implicit nature of the boundary conditions because the velocity 
and temperature from the $D B C s$ are computed in each pressure-velocity iteration loop (PIMPLE), thus improving the stability and accuracy. The current implementation allows coupling two 3D domains, two boundaries of the same 3D domain, or one 3D domain with a 0D pipeline. There are previous works devoted to coupling OpenFOAM with external codes ([24][25][26]), based on explicit methods, showing accurate solutions of test benchmarks. However, it is expected that the current semi-implicit implementation will ensure accuracy and robustness under a wide range of flow conditions.

Some significant limitations of the 0D modelling should be highlighted: First, only mean values are available for each variable such as the density, pressure, velocity, and temperature, among others. This could represent an obstacle to model some significant phenomena that takes relevance in nuclear safety such as thermal natural circulation, and siphon effects, among others. However, empirical models can be included to predict these phenomena in terms of inlet/outlet temperatures, pressures and elevations. Second, the inability to solve complex hydraulic networks having bifurcations and several cross section pipes. About the last, several components like heat exchangers present many section changes, but the final effect can be well represented with only one pressure drop coefficient[27]. .

Beyond the limitations, 0D momentum and energy equations are easier to be coded than 1D ones. Then, the current DBC gives a framework for future enhancing and implementation of dedicated models for specific applications, both in nuclear engineering as well as in many other industrial fields.

In the current paper, the coupling tool is briefly introduced before to carry on the simulation of the TRIGA MARK I IPR-R1 open-pool research reactor. To achieve the last, several thermal-hydraulics components such as pipes, tanks, pumps and heat exchangers, are included in the DBC. The reactor is simulated using a $3 \mathrm{D}$ CFD domain for the reactor pool and $0 \mathrm{D}$ modelling for the external coolant circuit. The 0D model considers the full pipeline including the pipes, elbows, pump, local flow restrictions, and the heat exchanger by condensing the pressure losses into an effective pressure loss coefficient. The heat exchanger is a typical shell-tubes device for which an empirical model is implemented. A few works have addressed the simulation of the pool water of TRIGA Mark II and TRIGA Mark III reactors using 3D CFD and system codes. However, studies using 3D-0D are not available in open literature. This model could be used to the assessment of reactor modifications both in the external loop systems as well as inside the pool. This work reports long-time simulation concerning to the reactor from start-up until to reach the steady-state at two core powers $(100 \mathrm{~kW}$ and $265 \mathrm{~kW}$ ), and the simulation of SBO and LOCA events. 


\section{GOVERNING EQUATIONS}

The computational model was implemented in the OpenFOAM-5.0 suite (Open Field Operation and Manipulation) [28], which is a free and open source code. The compressible solver "buoyantPimpleFoam" was employed. The linear Boussinesq approach was used to account for the dependency of the density with temperature. Other thermodynamic properties were considered constants. It should be remarked that the linear temperature approach is suitable for relatively low temperature variations less than $30^{\circ} \mathrm{C}$. Although this approach is widely used to simulate industrial problems, in high Rayleigh problems this could lead to significant errors not only due to the linear estimation of the density but also due to the use of constant viscosity properties [29]. Single-phase Newtonian flows are mathematically described by the continuity, momentum, and energy equation balances, which can be written in a conservative form as read

$$
\begin{gathered}
\frac{\partial \rho}{\partial t}+\frac{\partial\left(\rho u_{j}\right)}{\partial x_{j}}=0 \\
\frac{\partial\left(\rho u_{j}\right)}{\partial t}+\frac{\partial\left(\rho u_{j} u_{i}\right)}{\partial x_{j}}=-\frac{\partial p}{\partial x_{i}}+\frac{\partial}{\partial x_{j}}\left(\tau_{i j}+\tau_{t, i j}\right)+\rho g_{i} \\
\frac{\partial(\rho h)}{\partial t}+\frac{\partial}{\partial x_{j}}\left(\rho h u_{j}\right)-\frac{\partial p}{\partial t}=\frac{\partial}{\partial x_{k}}\left(\kappa_{e f f} \frac{\partial T}{\partial x_{k}}\right)
\end{gathered}
$$

where $g_{i}$ is the acceleration of gravity, $\tau_{t, i j}$ is the turbulent stress tensor, and $\tau_{i j}$ is the laminar stress tensor, which can be calculated by the following expression:

$$
\tau_{i j}=\mu\left[\left(\frac{\partial u_{i}}{\partial x_{j}}+\frac{\partial u_{j}}{\partial x_{i}}\right)-\frac{2}{3}\left(\frac{\partial u_{k}}{\partial x_{k}}\right) \delta_{i j}\right]
$$

As noted, the energy equation 3 is formulated in terms of the enthalpy $h$, where $\kappa_{e f f}=\alpha_{e f f} \rho C_{p}$ is the effective conductivity, and $C_{p}$ is the specific heat. The effective thermal diffusivity $\alpha_{e f f}$ is calculated in terms of the kinematic viscosity and the turbulent viscosity:

$$
\alpha_{e f f}=\frac{\nu_{0}}{P r}+\frac{\nu_{t}}{P r_{t}}
$$

In Equation $5 \mathrm{Pr}$ and $\mathrm{Pr}_{t}$ are the laminar and turbulent Prandtl numbers. In this work the $P r$ is assumed constant and equal to 0.7 .

A closure equation is required to estimate the turbulent viscosity and solve the RANS equations. In this work the realizable $k-\epsilon$ model [30] is choice. This introduces two additional transport equations, one for the turbulent kinetic energy $(k)$ (Eq:6), and the other for the turbulent dissipation rate $(\epsilon)$ (Eq: 7).

$$
\frac{\partial(\rho k)}{\partial t}+\frac{\partial\left(\rho k u_{i}\right)}{\partial x_{i}}=\frac{\partial}{\partial x_{j}}\left(\frac{\mu_{t}}{\tau_{k}} \frac{\partial k}{\partial x_{j}}\right)+2 \mu_{t} S_{i j} S_{i j}-\rho \epsilon
$$




$$
\frac{\partial(\rho \epsilon)}{\partial t}+\frac{\partial\left(\rho e u_{i}\right)}{\partial x_{i}}=\frac{\partial}{\partial x_{j}}\left(\frac{\mu_{t}}{\tau_{\epsilon}} \frac{\partial \epsilon}{\partial x_{j}}\right)+C_{1 \epsilon} 2 \frac{\epsilon}{\kappa} \mu_{t} S_{i j} S_{i j}-C_{2 \epsilon} \rho \frac{\epsilon^{2}}{k}
$$

where $S_{i j}$ is the rate of strain tensor, and $\mu_{t}$ is the turbulent dynamic viscosity, which is defined by the following equation:

$$
\mu_{t}=\rho C_{\mu} \frac{k^{2}}{\epsilon}
$$

The model constants are taken by default [31]:

$$
C_{\mu}=0.09 \quad C_{1 \epsilon}=1.44 \quad C_{2 \epsilon}=1.92 \quad \tau_{k}=1.00 \quad \tau_{\epsilon}=1.3
$$

\subsection{Zero-dimensional modelling}

In this section, the zero-dimensional momentum and energy balance equations are presented. First, the mathematical background for the 0D model is analysed. Then, the numerical aspects of $0 \mathrm{D} / 3 \mathrm{D}$ coupling are discussed, and finally the code implementation of the $D B C$ is introduced.

\subsubsection{Momentum equation}

The dimensional reduction of the momentum Equation 2 to the 0D form is achieved after a volumetric and temporal integration by applying the Backward-Euler temporal discretization and modelling the frictional and form pressure losses along all the pipeline through the Darcy-Weisbach approach. As a result, the $0 \mathrm{D}$ momentum equation takes the following form:

$$
\begin{aligned}
\left(\rho L S_{f}\right) \frac{U-U_{0}}{\Delta t} & =\left(p_{\text {inl }}-p_{\text {out }}\right) S_{f}+\rho g\left(h_{\text {inl }}-h_{\text {out }}\right) S_{f} \\
& +S_{f} \frac{|U|}{U}\left(\frac{1}{2} \rho \lambda \frac{L}{D_{h}} U^{2}+\frac{1}{2} \rho K U^{2}\right)+\Delta P_{\text {ext }}
\end{aligned}
$$

Note that Equation 9 is written in a scalar form. $p_{\text {inl }}, p_{\text {out }}, h_{\text {inl }}$, and $h_{\text {out }}$ are the pressures and elevations at the inlet and outlet ends of the pipeline. $S_{f}$ and $L$ are the cross-section and length of the pipeline, $\lambda$ is the friction coefficient and $K$ is the total form coefficient, which is calculated by summing all the components $\left(K=\sum_{i=1, n} K_{i}\right)$. That is, elbows, local contractions, and valves, among others. Finally, $D_{h}$ is the hydraulic diameter, which is the same for all the pipeline. The term $\rho L S_{f}$ in the left-hand side of the equation accounts for the inertial of the overall fluid along the circuit, while the last term $\Delta P_{\text {ext }}$ allow to include external pressure sources/sinks such as a pump. The quotient $|U| / U$ is need to recover the direction of the flow. The friction factor $\lambda$ for laminar flow $(R e<2200)$ is estimated by the Hagen-Poiseuille correlation, while for turbulent flow (Re $\geq 3000)$ the Colebrook-White equation [32] is applied. The external source in Equation 9 could be calculated as $\Delta P_{e x t}=\rho g H$ where $H$ is the pump head. 
The current implementation allows including a characteristic Head(H)-Flow Rate(Q) pump curve through three coefficients $\left(C_{1}, C_{2}\right.$ and $\left.C_{3}\right)$ describing a parabolic curve $H=f(Q)=C_{1} Q^{2}+C_{2} Q+C_{3}$. Currently, only the first pump quadrant can be included in the $D B C$.

As noted, the velocity in equation 9 depends basically on the end pressures and the velocity $U_{0}$ of the previous time step. The Figure 2 sketches the three possible coupling configurations: Figure 2-a is the simplest case in which the left side of the $3 \mathrm{D}$ domain is connected to a constant pressure $D B C\left(p_{i n l}=p_{0}\right)$, such as the atmospheric pressure, and the velocity imposed to the boundary condition $b c 1$ in the $3 \mathrm{D}$ model is $U_{b c 1}=f\left(t, p_{b c 1}, p_{0}\right)$. On the other hand, in Figure 2-b shows a $D B C$ used to model a tank. In this case the $D B C$ allows considering the time-variation of $h$ to estimate the flow velocity in the boundary condition $b c 1$ as $U_{b c 1}=f\left(t, p_{b c 1}, p_{i n l}\right)$, where $p_{i n l}$ is a simple expression considering the reference pressure $p_{0}$ plus the weight of water column, $p_{i n l}(t)=p_{0}+\rho g h(t)$, where $h(t)=h_{0}-\left(U_{b c 1} S_{f} \Delta t\right) / A_{t}$, and $A_{t}$ is the cross section area of the tank. Finally, a more general configuration is sketched in Figure 2-c. Here, two boundary condition of 3D domains ( $b c 1$ and $b c 2$ ) are coupled through a $D B C$ representing a $0 D$ pipeline. The velocity in each $3 \mathrm{D}$ patches depend on the pressures in both patches $\left(U_{b c 12}=f\left(t, p_{b c 1}, p_{b c 2}\right)\right)$.

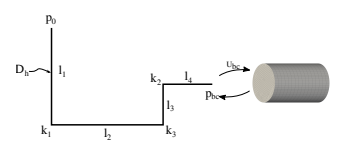

a)

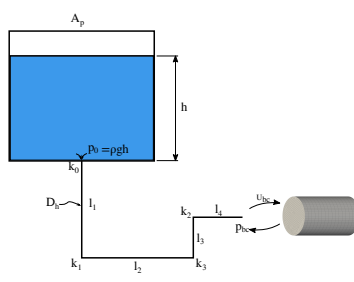

b)

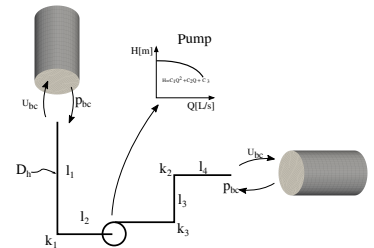

c)

Figure 2: Sketches of the three possible coupling configurations for the $D B C$ : a) A 3D domain connected to a 0D pipeline subjected to a constant pressure $P_{0}$, b) A 3D domain connected to a variable level tank, c) Two 3D domains joined through a $0 \mathrm{D}$ pipeline.

The time integration of the $U$ equation 9 takes the following form:

$$
\begin{aligned}
\mathrm{U}^{n}=U^{n-1}+\Delta t & {\left[\left(p_{b c, 1}^{n}-p_{b c, 2}^{n}\right) /(\rho L)+g\left(h_{\text {inl }}-h_{\text {out }}\right) / L\right] } \\
+\Delta t & \left(\frac{1}{2} \lambda^{n} \frac{L}{D_{h}}\left(U^{n}\right)^{2}+\frac{1}{2} K\left(U^{n}\right)^{2}\right) / L+\left(\Delta t H^{n} g\right) / L
\end{aligned}
$$

The superscripts $n$ and $n-1$ indicate the current and previous time steps, respectively. Considering that the boundary conditions are implicitly 
treated in OpenFOAM, the non-linear term corresponding to the pressure loss factor correlation and the pump head in Equation 10 is semi-implicitly solved because $\lambda$ and the pump head $H$ are updated with the current $U^{n}$ inside each PIMPLE loop. Therefore, the Equation 10 becomes a quadratic equation in terms of $U^{n}$, which can be also solved analytically inside the iteration loops:

$$
\mathbf{U}^{\mathbf{n}}=\frac{-B \pm\left(B^{2}-4 A C\right)^{1 / 2}}{2 A}
$$

where $A=\frac{1}{2} \Delta t\left(\frac{K_{E f f}}{L}+\frac{\lambda^{n}}{D_{h}}\right), B=1$, and $C=-U^{n-1}-\frac{\Delta t}{L}\left[\left(p_{b c 1}^{n}-p_{b c 2}^{n}\right) / \rho\right.$ $\left.+g\left(h_{\text {inl }}-h_{\text {out }}\right)+H^{n} g\right]$.

This has the constrain that the term $4 \mathrm{AC}$ in the root argument have to be less than $B^{2}$.

The velocity boundary condition is updated when the momentum matrix is assembled. The buoyantPimpleFoam solver use the PIMPLE algorithm for the pressure-velocity coupling. This semi-implicit method is a combination of PISO (Pressure Implicit with Splitting of Operator) and SIMPLE (SemiImplicit Method for Pressure-Linked Equations) algorithms [33]. Therefore, the $D B C$ is recalculated in each loop iteration, taking the upgraded values of the pressure at the boundaries, before to solve the momentum equation.

\subsubsection{Thermal equation}

The heat exchanger in the pipeline is modelled through an additional thermal boundary condition $(T B C)$, which takes into account the heat transfer between the primary and secondary circuits. The current implementation consist on a generic shell and tubes heat exchanger as shown in Figure 3 . This considers the coolant flow rate $\left(\dot{m}_{h}\right)$ and temperature $\left(T_{h, \text { inl }}\right)$ coming from the reactor as well as the mass flow $\left(\dot{m}_{c}\right)$ and temperature $\left(T_{c, i n l}\right)$ at the inlet of the secondary circuit.

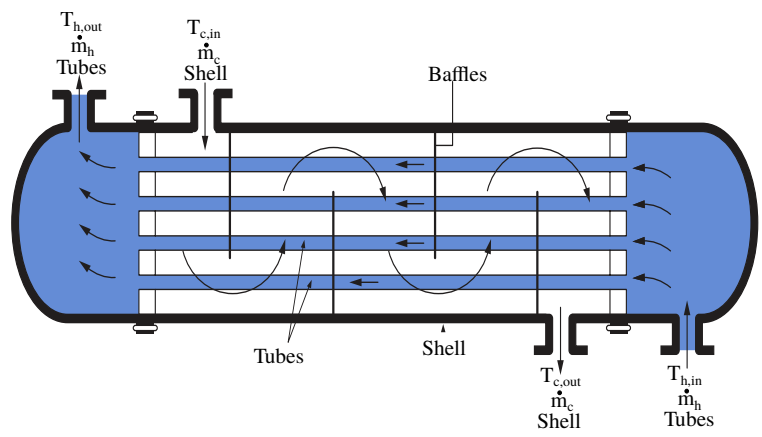

Figure 3: Sketch of the heat exchanger

The outlet temperatures $T_{h, \text { out }}$ and $T_{c, \text { out }}$ are given from the widely used Effectiveness-NTU Method, which is summarised in Table 1. This method 


\begin{tabular}{cc}
\hline Equation & Equation Number \\
\hline$\epsilon=\frac{\dot{Q}}{\dot{Q}_{\max }}$ & $1 . \mathrm{a}$ \\
$\dot{Q}=C_{c}\left(T_{c, \text { out }}-T_{c, \text { inl }}\right)=C_{h}\left(T_{h, \text { inl }}-T_{h, \text { out }}\right)$ & $2 . \mathrm{a}$ \\
$\Delta T_{\max }=T_{h, \text { inl }}-T_{c, \text { inl }}$ & $3 . \mathrm{a}$ \\
$\dot{Q}_{\max }=C_{\min }\left(T_{h, \text { inl }}-T_{c, \text { inl }}\right)$ & $4 . \mathrm{a}$ \\
$c=\frac{C_{\min }}{C_{\operatorname{mgx}}} A_{s}$ & $5 . \mathrm{a}$ \\
$N T U=\frac{U_{\text {gl }} A_{\text {min }}}{C^{2}}$ & $6 . \mathrm{a}$ \\
$\epsilon=2\left\{1+c+\sqrt{1+c^{2}} \frac{1+\exp \left[-N T U \sqrt{1+c^{2}}\right]}{1-\exp \left[-N T U \sqrt{1+c^{2}}\right]}\right\}^{-1}$ & $7 . \mathrm{a}$ \\
$T_{h, \text { out }}=T_{h, \text { inl }}-\frac{\dot{Q}}{C_{h}}$ & $8 . \mathrm{a}$ \\
\hline
\end{tabular}

Table 1: Summary of the Effectiveness-NTU Method

is based on a dimensionless parameter called the heat transfer effectiveness $\epsilon$ defined in Equation 1.a. Here, $\dot{Q}$ is the heat transfer rate, which can be determined from a thermal balance over the secondary or primary fluids, as shown in Equation 2.a. In Equation 2.a. $C_{c}=\dot{m}_{c} C p_{c}$ and $C_{h}=\dot{m}_{h} C p_{h}$, where $\mathrm{Cp}$ is the heat capacity. On the other hand, $\dot{Q}_{\max }$ is the maximum heat transfer rate (see Equation 4.a), which is determined based on the maximum temperature difference $\Delta T_{\max }$ from Equation 3.a. In Equation 4.a $C_{\min }$ is the smaller value between $C_{h}$ and $C_{c}$. Then, $c$ and $N T U$ are given by Equations 5.a and 6.a, where $U$ is the overall heat transfer coefficient. For a shell and tube heat exchanger, the effectiveness $\epsilon$ for one shell pass and four tube passes is computed by Equation 7.a [34]. Once $\epsilon$ and $\dot{Q}_{\max }$ are calculated, the heat transfer rate $\dot{Q}$ can be upgraded from Equation 1.a. Finally, the outlet temperature of the primary circuit $T_{h, \text { out }}$ is calculated through Equation 8.a. The outlet temperature of the secondary circuit can be also obtained from $\dot{Q}$.

The heat exchanger efficiency depends on the geometry as well as the mass flow rates and the temperatures of both systems. Therefore, each heat exchanger has a different efficiency, which typically involves two dimensionless groups: the capacity ratio $c$ and the number of transfer units $N T U$. In the Effectiveness-NTU method the heat transfer area $A$, the mass flow rates $\dot{m}_{h}=\dot{m}_{c}=\dot{m}$, the global heat transfer coefficient $U_{g l}$, and the inlet temperatures $T_{h, i n}$ and $T_{c, i n}$ are known. On the other hand, the unknown variables are the heat transfer rate $\dot{Q}$ and the outlet temperatures $T_{h, o u t}$ and $T_{c, \text { out }}$.

The temperature in the $T B C$ (Equation 8.a in table 1) is calculated in each PIMPLE iteration loop in similar way that the velocity in equation 10 to coupling the boundaries $b c 1$ and $b c 2$ : 


$$
T_{b c 1}^{n}=T_{b c 2}^{n}+\frac{q^{n}}{\rho C_{p} U^{n} S_{f}}
$$

where $T_{b c 2}$ is the temperature in the outlet boundary of the $3 \mathrm{D}$ domain, and $T_{b c 1}$ is the temperature computed by the $T B C$ and applied to the inlet boundary $b c 1$. The term $q$ represents the power transferred and is calculated by means of $\dot{Q}$. The energy equation matrix is assembled at the end of each PIMPLE loop, giving a semi-implicit coupling between velocity and temperature. The $T B C$ is recalculated previous to the energy equation matrix assembly in each PIMPLE loop.

The source files for the $D B C$ follow the general rules of all boundary conditions in OpenFOAM. That is, a source file, e.g. couplingPipeFvPatchVectorField.C, is created with the $\mathrm{BC}$ constructor/destructor. For the $D B C$ a fixed velocity value (Dirichlet) is imposed. Therefore, the function updateCoeffs() upgrades the velocity in each PIMPLE loop. Additionally, the declaration file is created, e.g. couplingPipeFvPatch VectorField.H. Here all constructors, variables and functions of the class are declared. More details about this procedure are available in the OpenFOAM BC sources (\$FOAM_SRC/finiteVolume/fields/fvPatchFields).

Once the source code is compiled, the $D B C$ can be used for any singlephase solver in OpenFOAM. The user only has to define the $D B C$ parameters in the model input file of the initial directory $(0 / U$ and $0 / T$ files $)$. A basic $D B C$ setting for the hydraulic coupling of two boundaries (e.g. INL and OUT) is depicted in the listing 1:

Listing 1: Setting of parameters of the library couplingPipeFixedValue.

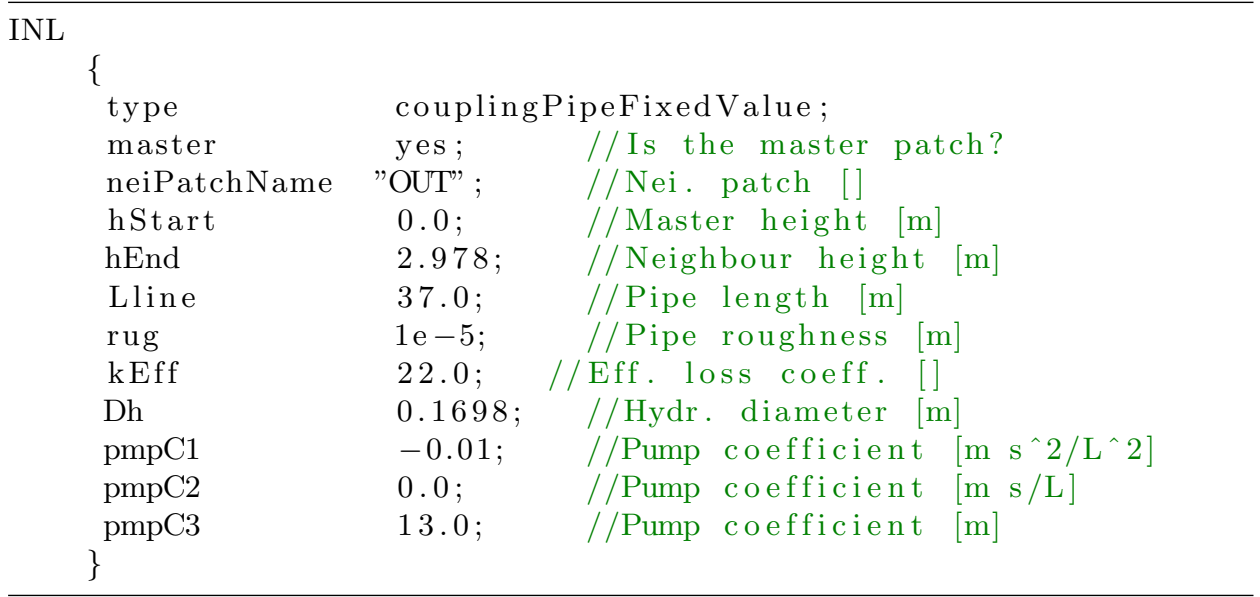

The $D B C$ showed in Listing 1 corresponds to the pipeline linking the patches "INL" and "OUT". This input data example also contains the pump $\left(H(Q)=-0.01 Q^{2}+13.0\right)$, and the inlet/outlet height difference $(h E n d-$ $h$ Start $=2.978)$. The pipe has the length Lline of $37.0 \mathrm{~m}$, the hydraulic 
diameter $D h$ of $0.1698 \mathrm{~m}$, and the roughness rug of $1 \times 10^{-5} \mathrm{~m}$. Finally, the form losses are condensed in the Effective loss coefficient $k E f f$ of 22 .

Some model limitations can be mentioned; The main one is the use of a single pipe section. But, the current $D B C$ could be enhanced to account for different pipe sections, obtaining the local frictional coefficients for the corresponding velocities in each pipe stretch. The use of $0 \mathrm{D}$ models for solving multiple-section pipelines is not new [2]. In fact, the implementation of codes to solve more complex pipelines with bifurcations or several loops interconnected could be difficult, but not impossible. However, these code capabilities are not required for the TRIGA Mark-I simulation. Other limitation of the current model is that the pump inertia is not considered. In this sense, the coast-down model proposed by Hong et al. [35] could be applied. This considers the pump shaft velocity $(\omega)$, which achieves from the angular momentum equation of pump shaft $\left(\sum_{i} \tau_{i}=I d \omega / d t\right.$, where I is the inertia, and $\sum_{i} \tau_{i}$ is the torque summation). Finally, the fluid momentum and the shaft angular momentum are coupled with the Uddin method [36] considering that the pump head is proportional to the square of the pump speed. However, the pump of the TRIGA reactor is small and the coast-down effect can be neglected.

\section{Results}

In this section the steady state for two core power conditions is presented. After that, the $S B O$ and large $L O C A$ events are simulated in order to study the most severe cases from safety point of view.

In order to reduce the complexity of the 3D domain the internals of the pool were broadly simplified, and a uniform volumetric heat source was imposed in the core. Figure 4 shows the 3D computational domain and the scheme of the cooling circuit represented by the $D B C$ and $T B C$. The Table 2 resumes the main parameters of the model.

The pool domain was meshed with a hybrid mesh (840,037 cells). For steady-state simulations the pool level keeps constant and single-phase simulations were performed. The free surface in contact with the atmosphere is represented as a slip wall with an external thermal boundary condition to account for the heat transfer. To achieve that a constant coefficient $h_{\text {free }}$ of $115\left[\mathrm{~W} / \mathrm{m}^{2} \mathrm{~K}\right]$ was obtained from literature [37]. The rest of the pool walls and the pool floor were assumed as adiabatic, which is justified by the thick concrete wall surrounded the pool.

The simulation of large transients considering the full geometry of the core is not possible with the current computational resources and the RANS turbulence models give excessive errors in the pressure loss estimation of complex internal flows. Due to that, the pressure drop across the core was modelled as a Forchheimer porous media: 


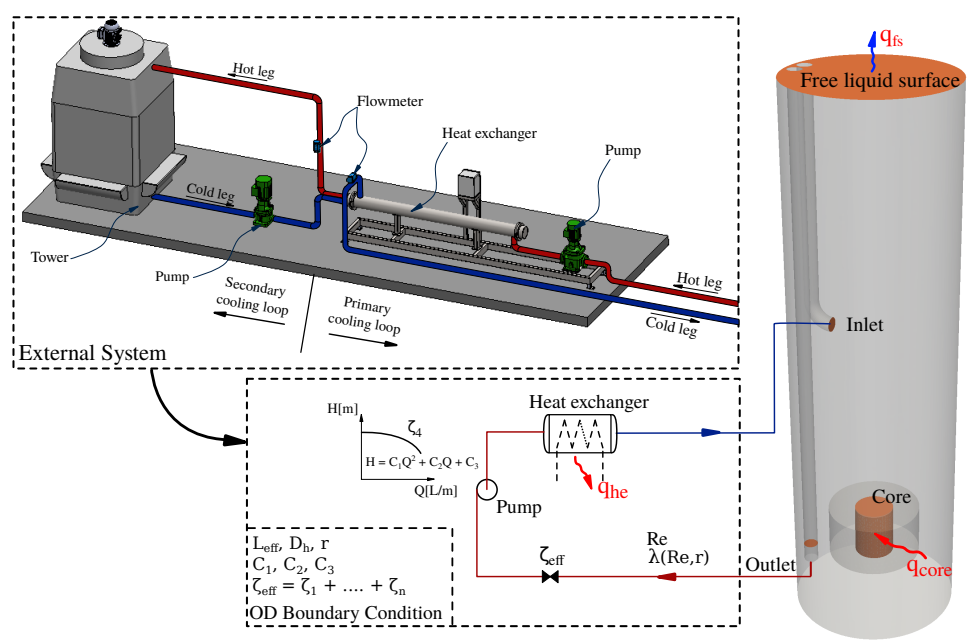

Figure 4: Details of the problem domain.

$$
\nabla p=F \frac{\rho}{2} u^{2}
$$

where $F=60.3$ is the Forchheimer coefficient, which was estimated from the data reported by Mesquita et al. [38], who measured the temperature and velocity at five locations at the bottom (inlet) and the top (outlet) sides of the core with a core power of $265 \mathrm{~kW}$. From these data, we obtained the inlet and outlet flow-weighted average temperatures and the mean mass flow rate was calculated from a simple thermal balance $\left(\dot{m}=\frac{\dot{Q}}{C_{0} \Delta T}\right)$. After that, the 3D CFD model was run for a set of Forchheimer coefficients until to find the best fitting. It should be noted that the Forchheimer coefficient was defined in terms of a particular steady-state condition. This could not be right for the accident scenarios. But, information about the internal core flow in SBO and LOCA events is unavailable.

\subsection{Steady-state}

The nominal heat power of the TRIGA Mark I reactor is $100 \mathrm{~kW}$, but it can be operated with higher powers. For instance, Mesquita et al. [38] carried out experimental tests for the high power of $265 \mathrm{~kW}$. Therefore, steady state simulations at both powers were performed.

The steady state condition was reached once the average temperature of the reactor pool reached a constant value after more than 76,000 s. The calculus was performed in parallel computing with a distributed memory architecture using 24 processors (E5-1660-v3, 26 GB-RAM DDR3, 160GBHD, Infiniband QDR4 Gbps) in the cluster Seshat belonging to CIMEC. More than $3 \mathrm{hrs}$ were needed to compute $1 \mathrm{hr}$ of real time. This time rate was 


\begin{tabular}{|c|c|}
\hline \multicolumn{2}{|l|}{ Primary cooling loop } \\
\hline Parameter & Value \\
\hline Total length $\quad[\mathrm{m}]$ & 37.0 \\
\hline$\zeta_{\text {tot }}[-]$ & 2001.2 \\
\hline Pump coef. $C_{1} \quad\left[m s^{2} / L^{2}\right]$ & -0.01 \\
\hline Pump coef. $C_{2} \quad[m s / L]$ & 0.0 \\
\hline Pump coef. $\mathrm{C}_{3} \quad[m]$ & 13.0 \\
\hline Roughness $[\mu m]$ & $1 \times 10^{-6}$ \\
\hline$\dot{m}[\mathrm{~kg} / \mathrm{s}](S-S$ target $)$ & 7.8 \\
\hline \multicolumn{2}{|l|}{ Secondary cooling loop } \\
\hline Parameter & Value \\
\hline Heat transfer area $\left[\mathrm{m}^{2}\right]$ & 47.0 \\
\hline$\dot{m}[\mathrm{~kg} / \mathrm{s}]$ & 11.1 \\
\hline $\mathrm{T}_{\text {in }}[K]$ & 299.8 \\
\hline$U_{g l} \quad[-]$ & 836.8 \\
\hline
\end{tabular}

Table 2: Problem parameters.

many times greater than the required with system codes, but faster than the expected with full 3D models.

Table 3 resumes the computational results and the experimental data. The Case 1 refers to the design power of $100 \mathrm{~kW}$, while Case 2 corresponds to the largest power of $265 \mathrm{~kW}$, for which some experimental data are available. Despite of the notorious simplification of the core geometry, the porous media leads to a good agreement in terms of the mass flow rate and the temperature increment $\Delta T$ across the core. The mass flow rate in the external circuit is well fitted by the $D B C$. As noted, the pool heated up around $10 \mathrm{~K}$ more by increasing the power from $100 \mathrm{~kW}$ to $265 \mathrm{~kW}$. The larger core power increases the buoyant phenomena, and the core mass flow rate suffers a significant increment from $3.4 \mathrm{~kg} / \mathrm{s}$ ups to $4.7 \mathrm{~kg} / \mathrm{s}$. This improves the core cooling efficiency. That is, the power increases 2.65 times while the temperature increment $\Delta T$ grows 1.91 times. Despite of the significant thermal stratification along the vertical direction, the bulk temperature is only a little higher than the outlet temperature. This is due to the location of the outlet mouthpiece in the bottom side of the pool.

Figure 5 displays the bulk temperature $\left(T_{b u l k}\right)$, the power transferred through the free surface and the heat exchanger. Results corresponds to Case $2(265 \mathrm{~kW})$ during a time windows of $6000 \mathrm{~s}$ after the steady-state is reached. As noted, the $T_{b u l k}$ fluctuated around $315 \mathrm{~K}$ with variations less than $0.05 K$. The power through the free surface also holds quite constant 


\begin{tabular}{|l|c|c|c|}
\hline \multicolumn{4}{|c|}{ Pool reactor and External circuit } \\
\hline Parameter & Case 1 & \multicolumn{2}{|c|}{ Case 2 } \\
\hline- & $C F D_{100 k W}$ & $C F D_{265 k W}$ & $\operatorname{Exp}_{265 k W}[38]$ \\
\hline Power $[\mathrm{kW}]$ & 100 & 265 & 265 \\
$\dot{m}[k g / s]$ & 7.95 & 7.94 & 7.8 \\
$\mathrm{~T}_{\text {bulk }}[K]$ & 305.85 & 315.15 & - \\
$\mathrm{T}_{\text {inl }}[K]$ & 302.54 & 306.46 & - \\
$\mathrm{T}_{\text {out }}[K]$ & 305.53 & 314.26 & - \\
\hline \multicolumn{4}{|c|}{ Core } \\
\hline$\dot{m}[k g / s]$ & 3.4 & 4.7 & 4.42 \\
$\mathrm{~T}_{\text {core }}[K]$ & 309.46 & 321.7 & - \\
$\mathrm{T}_{\text {inl }}[K]$ & 305.72 & 314.5 & - \\
$\mathrm{T}_{\text {out }}[K]$ & 312.72 & 327.9 & - \\
$\Delta T[K]$ & 7.0 & 13.4 & 14.3 \\
\hline
\end{tabular}

Table 3: Mean results.

around $5 k W$. This represents less than $2 \%$ of the core power. Therefore, the convection through the free surface is far to be enough to dissipate the core power during a $S B O$ event.

As noted in Table 3, the temperature differences among inlet and outlet inside the pool are more than $3 K$ and $8 K$ for Cases 1 and 2 respectively. In this context, the little variations in the bulk temperature displayed in Figure 5-a are 80 times lower than it. This temperature variations are clearly visualized in Figures 6 -a, and 6-b over vertical and horizontal cross planes. The results correspond to a time of $76,000 \mathrm{~s}$. The flow pattern is depicted by a central ascending plume reaching to the upper side of the pool. The maximum velocities are around $20 \mathrm{~mm} / \mathrm{s}$ close to the core outlet. The hot plume is $4 K$ hotter than the rest of the tank. The top half of the pool shows mean and maximum temperatures around $315 K$ and $317 K$, whereas the minimum temperature at the bottom half of the pool is around $313.2 \mathrm{~K}$. Over the inlet elbow, the temperature shows a homogeneous distribution (see Figure 6-a). The influence of the cold inlet jet at the middle of the pool is clearly observed in the cutting plane placed above to the inlet elbow. The cold jet intersects the ascending plume promoting a strong mixing. The inlet jet modifies the natural convection pattern caused by the core heating. Due to the incoming velocity the jet impacts to the right wall and splits into ascending and descending flows. At the upper half of the pool the flow ascends close the walls and descends through the rest of the cross section. Despite of the results are time-averaged of the fields, an asymmetric flow 
pattern is caused because the the inlet and outlet mouths are not in the same vertical plane.

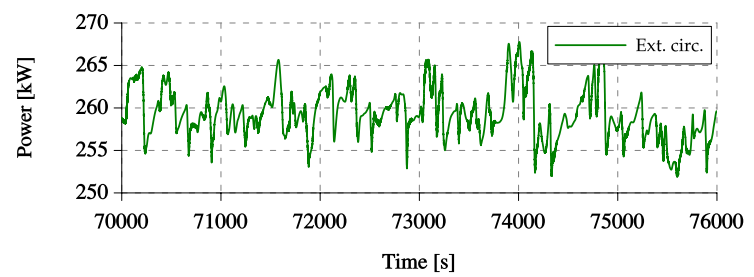

c)

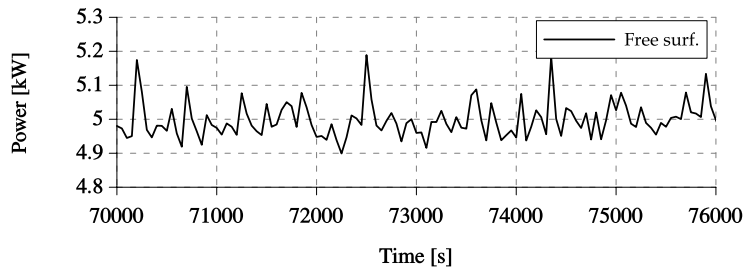

b)

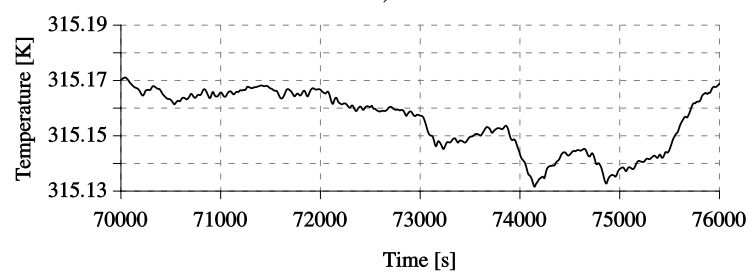

a)

Figure 5: Steady-state results for Case $2(265 k W)$. a) Bulk temperature. b) Power transferred through the free surface. c) Power transferred through the heat exchanger.

Case 1 presents a similar behaviour than Case 2 in terms of flow distribution. The main difference is the higher core outlet temperature. Despite the relatively coarse mesh used for the pool, the results display the main flow structures in the pool of TRIGA Mark I reactor. This detailed flow visualization, displaying the interaction between the hot ascending plume and the cold inlet flow, the non-symmetry flow distribution, the heat stratification and the overall mass flow through the core for different core powers is not possible by system code simulation.

\subsection{Station Black Out (SBO)}

The $S B O$ event for a constant core power of $265 \mathrm{~kW}$ is presented in this section. The event was initiated from the steady state and the pump shutdown was modelled by set to zero the pump coefficients in the $D B C$. As above mentioned, the motor-pump inertia and the pressure loss of the 


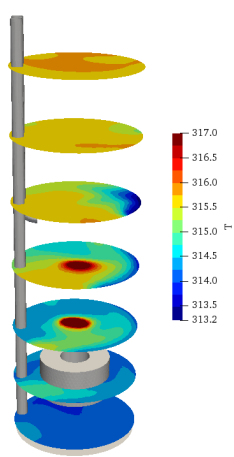

a)

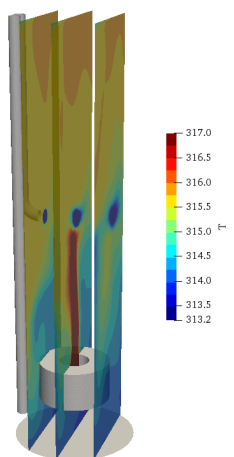

b)

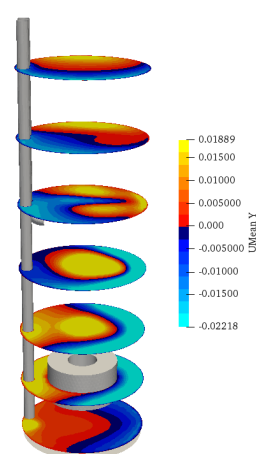

c)

Figure 6: Steady-state results for Case 2 (265kW). a)

Temperature in horizontal cross planes, b) Temperature in vertical cross planes. c) Vertical velocity in horizontal cross planes.

stopped pump were not considered in the current implementation of the $D B C$. The $S B O$ event also includes the shutdown of the secondary-circuit pump. Despite the fact that the external circuit has no effect on the behaviour of the pool, the present study is of great importance from the safety point of view.

The $S B O$ was simulated during $5000 \mathrm{~s}$. The Figure 7 shows the temporal evolution of the main parameters. At the beginning, the mass flow in the coolant circuit decreases quickly and the heat transfer is drastically reduced while the flow through the core displays a small reduction from the steadystate value of $4.7 \mathrm{~kg} / \mathrm{s}$ up to $4.2 \mathrm{~kg} / \mathrm{s}$ in the firsts $1000 \mathrm{~s}$ (see Figure 7a). During this period takes place the transition from forced to natural circulation through the core. Beyond $1000 \mathrm{~s}$ a slow, but continuous mass flow reduction through the core, is observed, although this remain over 4.1 $\mathrm{kg} / \mathrm{s}$ during the first $4500 \mathrm{~s}$. That is, the flow across the core reduced less than $10 \%$ during the first $4500 \mathrm{~s}$ while the core temperature increases more than $15 K$.

Figure 7-b displays the mean temperature of the pool (volume average in all the pool) and the mean temperature of the core. As noted, the core is around $6^{\circ} \mathrm{K}$ hotter than the rest of the pool. The overall heating rate is around $12.5 \mathrm{~K} / \mathrm{h}$ approximately, which is in agreement with the analytical solution also included in the graphic. The core temperature follows the same linear behaviour (with the same heating rate of $12.5 \mathrm{~K} / \mathrm{h}$ ).

Figure 7-c shows the power transferred across the free surface, which is the unique heat sink in this event. During the first $5000 \mathrm{~s}$ after the SBO 


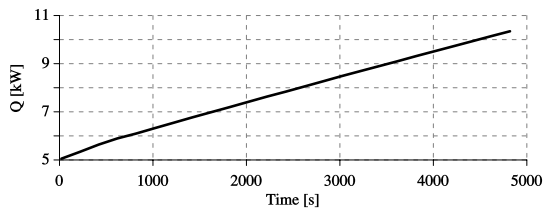

c)

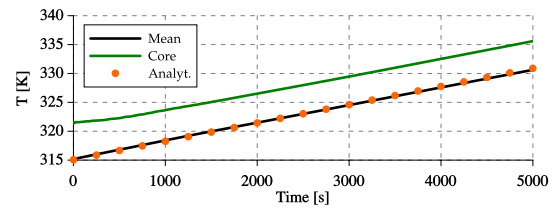

b)

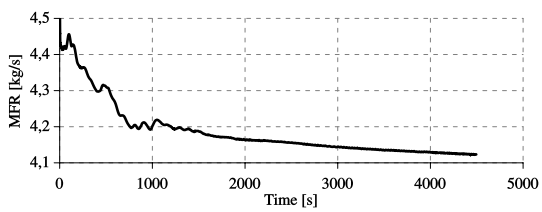

a)

Figure 7: Loss of Forced Convection at $265 \mathrm{~kW}$. a) Mass flow rate through the core, b) Mean temperatures in the core and reactor pool, c) Removed heat power through the free surface. 
event the power increases from $5.5 \mathrm{~kW}$ ups to $11 \mathrm{~kW}$, which represents $4 \%$ of the total core power. The heat transferred to the atmosphere is not enough to cool down the pool for a long time, causing a progressive heating of the core and the whole pool.

Figure 8 shows the temperature over the mid vertical plane at four times. The loss of forced flow reduces the pool mixing and promotes a large thermal stratification in the vertical direction. Inside the core the coolant flow from the bottom to the top is driven by natural circulation. The transition regime between the forced circulation to natural circulation is well organized and stable, thus guarantying the core cooling during this stage. The temperature of the coolant entering to the core progressively increases because of the pool is heating. In pictures, this temperature is $315 K$ at the beginning and increases to $317.6 K, 322 K$ and $327 K$ at $1600 \mathrm{~s}, 3200 \mathrm{~s}$ and $4800 \mathrm{~s}$, respectively.

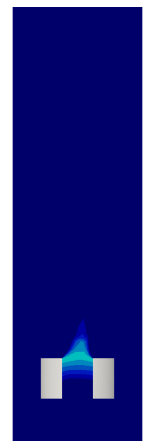

$\mathrm{t}=0 \mathrm{~s}$

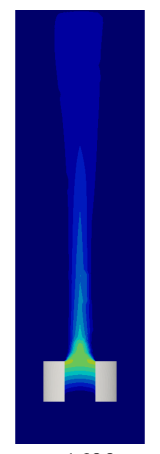

$t=1600 s$

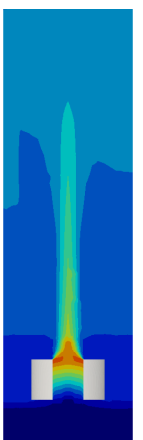

$\mathrm{t}=3200 \mathrm{~s}$

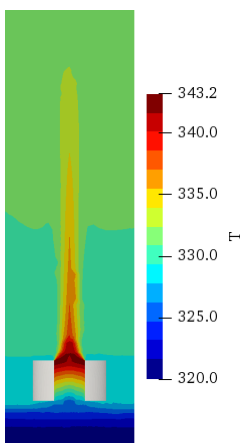

$\mathrm{t}=4800 \mathrm{~s}$

Figure 8: Loss Of Forced Cooling results: Temperature in the mid vertical plane at different times.

The core cooling is guarantee during all transient simulation and overheating zones are not observed. The natural convection and the thermal stratification help to keep the core cooled. This also improves the natural circulation inside the core. Natural circulation inside the external circuit is not expected because the heat transfer is missing. Moreover, the normal flow circulation should be invert overcoming the large pressure drop of the circuit.

\subsection{Loss Of Coolant Accident (LOCA)}

A large 2A-LOCA (double-ended break) downstream of the pump and upstream of the heat exchanger (See Figure 1) for the core power of $100 \mathrm{~kW}$ was simulated. Clearly, the break location represented the worst situation in terms of the loss of coolant. Moreover, the pump continued to run all the time quickly draining the pool water. Due to the break location and 
the fact the coolant circuit is elevated from the pool surface, a loss of water through the cold leg of the circuit is not expected neither by gravity force not by siphon effect. Due to the circuit layout, others break locations seems not to be relevant. Negligible water leakage is expected for any break placed upstream of the pump. In the other hand, the mass flow through a break placed downstream of the heat exchanger should be similar than the mass flow during normal operation because the most of the pressure drop takes place across the heat exchanger. Therefore, the studied large $L O C A$ represents the worse loss of water condition.

Attending to that, the large $L O C A$ event was simulated by using a $D B C$ to connect the pool outlet patch with a fixed atmospheric pressure condition. For the inlet patch a wall boundary condition was imposed. In this particular case the pool drainage was tracked using the Volume Of Fluid $(V O F)$ method with the compressibleInterFoam solver. The simulation was carried out for $450 \mathrm{~s}$, which was enough to reach the core uncovering.

The main relevant results of the large $L O C A$ are displayed in Figure 9. Figure 9-a shows the mass flow through the break. The water leakage fast increases from the nominal value of $7.8 \mathrm{~kg} / \mathrm{s}$ ups to $35 \mathrm{~kg} / \mathrm{s}$ in less than one second. This is mainly due to the sudden loss of pressure drop of the heat exchanger, which causes an increase on the flow pumped in agreement with the characteristic pump curve set in the $D B C$. The flow reaches a peak value before drops slowly due to the progressive reduction of the pool level. The pool inventory is displayed in Figure 9-b. This diminishes almost linearly, and around 13,000 $\mathrm{kg}$ of water are drained from the pool in only $450 \mathrm{~s}$. The loss of inventory and the constant core power causes a slow pool temperature increment of $1 K$ during the simulation, as shown in Figure 9-c. The exponential heating is due to the continuous pool emptying. The mass flow through the core is displayed in Figure 9-d. Some interesting features can be pointed out: the steady-state mass flow of $3.4 \mathrm{~kg} / \mathrm{s}$ is drastically reduced below to $1 \mathrm{~kg} / \mathrm{s}$ during the first second and remains below to 0.5 $\mathrm{kg} / \mathrm{s}$ during the first $130 \mathrm{~s}$. After that, the flow through the core inverts from upward to downward direction doe to the strong suction in the outlet throttle. The downward flow holds almost constant around $-0.5 \mathrm{~kg} / \mathrm{s}$ until the core is uncovered and the circulation of the water is interrupted. The core temperature is displayed in Figure 9-e. The core heat up from $310 \mathrm{~K}$ to $340 \mathrm{~K}$ during the first $300 \mathrm{~s}$ and then the temperature remains almost constant in agreement with the almost constant mass flow through the core. Unlike the $S B O$, for which the flow through the core is slightly reduced guarantying the core cooling, in the large $L O C A$ event the core mass flow rate is drastically reduced and the core temperature increases more than $33 \mathrm{~K}$ during the first $300 \mathrm{~s}$.

Figure 10 helps to understand the changes on the coolant patterns during the pool drainage. The velocity scale range was bounded to $0.05 \mathrm{~m} / \mathrm{s}$ to better visualisation of the low velocity zones. The first picture corresponds 


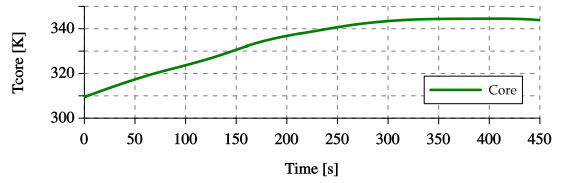

e)

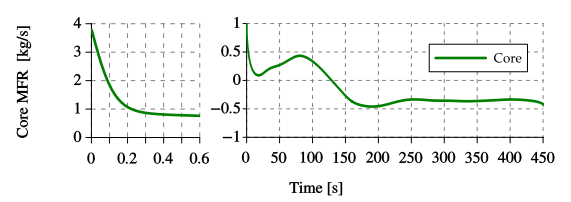

d)

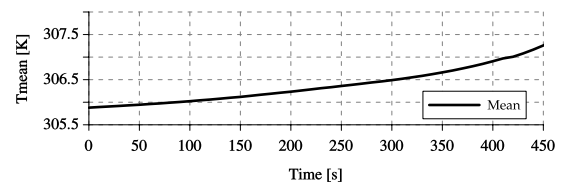

c)

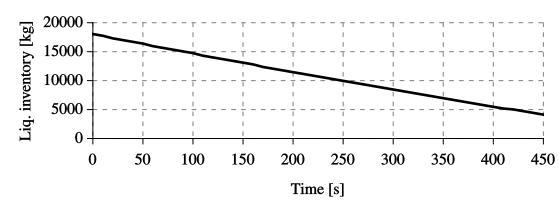

b)

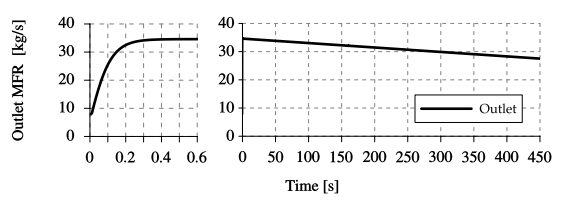

a)

Figure 9: Loss of Coolant Accident. a) Mean pool temperature, b) Mean core temperature, c) Mass flow rate through the break, d) Mass flow rate through the core, e) Pool inventory 


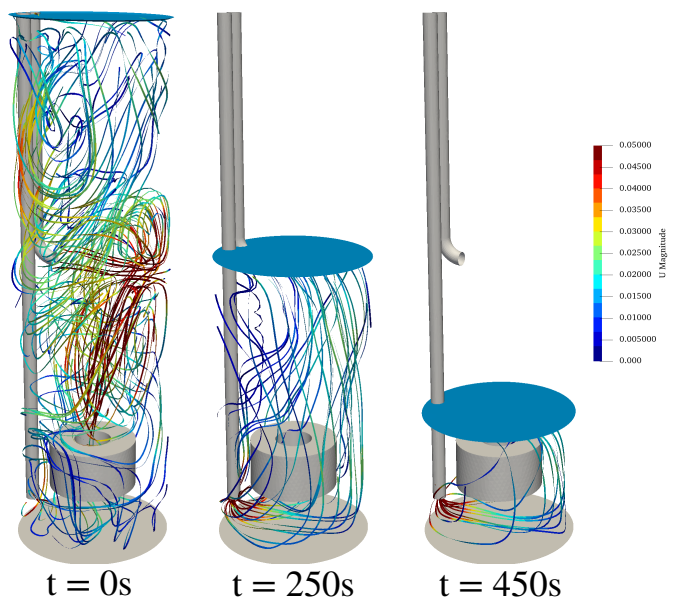

\section{Figure 10: Loss of Coolant Accident. Pool level evolution and streamlines at $0 \mathrm{~s}, 250 \mathrm{~s}$, and $450 \mathrm{~s}$.}

to the steady-state condition before the $L O C A$. In this time the ascending plume rising from the core to the middle of the pool is clearly depicted. The plume is broken by the jet coming from the inlet elbow. In the second picture, corresponding to $250 \mathrm{~s}$, the pool level drops reaching the middle of the pool. The streamlines allow to observe the quite uniform descending flow with most of the coolant passing not across the core but around this. Due to the location of the outlet throttle, the flow descends predominantly over the wall opposite to the outlet. Finally, at $450 \mathrm{~s}$ the pool level is close to the top of the core and the circulation of water through the core is not possible.

The current model allows predicting the time need to achieve the core uncovering. The postulated accident keeping the pump running represents an extreme situation. In a real situation it should be expected that the pump shut down occurs once the pool level reaches a safety set point value. After that, the loss of water through the break should be quickly reduced.

\section{CONCLUSIONS}

This paper addressed with the simulation of steady-state and postulated accident events of the open-pool reactor TRIGA MARK I -IPR1. A multidimensional model was reached by coupling a 3D domain of the pool reactor and $0 \mathrm{D}$ modelling of the external coolant circuit. The last was able through the implementation of dynamic boundary conditions in the OpenFOAM suit. Thermal-hydraulic components such as tanks, pipes, elbows, flow restrictions, pumps, and heat exchangers to represent a loop were coded for this particular case. 
The steady-state simulations for the normal operation core power of $100 \mathrm{~kW}$ and for the higher power of $265 \mathrm{~kW}$ were performed. The core cooling efficiency was assessed in both cases. At the higher power the mean temperature of the core incremented from $309 K$ to $321 K$, improving the natural circulation through the core. The mass flow grew from $3.4 \mathrm{~kg} / \mathrm{s}$ up to $4.7 \mathrm{~kg} / \mathrm{s}$ helping the core cooling. Two sensible accident events were evaluated: the SBO event for a full power of $265 \mathrm{~kW}$ was simulated to verify the long-time coolant capacity by natural convection inside the pool. A slightly reduction of the mass flow rate from $4.7 \mathrm{~kg} / \mathrm{s}$ up to $4.1 \mathrm{~kg} / \mathrm{s}$ was found in the core. However, the mean temperature of the core incremented from $321 \mathrm{~K}$ up to $335 \mathrm{~K}$ not only due to the mass flow reduction but also due to the heating of the overall pool. During this time the heat transfer through the free surface increased from $5.5 k \mathrm{~W}$ up to $11 \mathrm{~kW}$, although this power remained less than $2 \%$ of the core power. The pool heating rate predicted by the computational model was in accordance with analytical data and the macroscopic thermal balance. Despite of that, the removal of heat in the core was enough to guarantee the core safety for more than $5000 \mathrm{~s}$. Finally, a large Loss Of Coolant Accident (LOCA) for a core power of $100 \mathrm{~kW}$ and keeping the pump running was simulated. The results allow find that, under this extreme condition, the pool is quickly empty in less than 500s. During the first $130 \mathrm{~s}$ the mass flow through the core reduced to less than $1 \mathrm{~kg} / \mathrm{s}$ and the flow inverts from the upward direction to the downward one. After that the mass flow remains almost constant around $0.5 \mathrm{~kg} / \mathrm{s}$. The core is uncovered after $450 \mathrm{~s}$ and the core cooling is not possible.

\section{Acknowledgements}

The authors would like to thank Universidad Nacional del Litoral (CAI+D 2016 PIC 50420150100067LI), Agencia Nacional de Promocion Cientifica y Tecnologica ANPCyT (PICT 2016-2908) and Consejo Nacional de Investigaciones Cientificas y Tecnologicas (CONICET). The authors wish to acknowledge also the Universidade Federal de Minas Gerais. 


\section{References}

[1] N. E. Todreas, M. S. Kazimi, Nuclear systems: Vol. 1, Francis and Taylor.

[2] E. E. Lewis, Nuclear power reactor safety.

[3] C. Fletcher, R. Schultz, Relap5/mod3 code manual volume v: User's guidelines, Idaho National Engineering Laboratory, Lockheed Idaho Technologies Company, Idaho Falls, Idaho 83415.

[4] H. Lerchl, G.Austregesilo, Athlet mod 2.1 cycle a users manual, Gesellchaft für Anlagen-und Reaktorsicherheit (GRS) mbH.

[5] H. Xie, S. He, The scdap/relap5 3.2 model of ap1000 on sbloca, Progress in Nuclear Energy 61 (2012) 102-10\%.

[6] F. De Rosa, C. Lombardo, F. Mascari, M. Polidori, P. Chiovaro, S. D'Amico, I. Moscato, G. Vella, Analysis of a station black-out transient in smr by using the trace and relap5 code, in: Journal of Physics: Conference Series, Vol. 547, IOP Publishing, 2014, p. 012035.

[7] A. Prošek, L. Cizelj, Long-term station blackout accident analyses of a pwr with relap5/mod3. 3, Science and Technology of Nuclear Installations 2013.

[8] S. F. Corzo, M. Guala, A. Lazarte, Modelo de la central nuclear embalse con relap5: Accidente de pérdida de refrigerante en la tubería de aspiración de una de sus bombas, in: XXIII Congreso de Métodos Numéricos y sus Aplicaciones (ENIEF), La Plata, 2017.

[9] A. Mesquita, A. Costa, C. Pereira, M. Veloso, P. Reis, Experimental investigation of the onset of subcooled nucleate boiling in an openpool nuclear research reactor, in: Film and Nucleate Boiling Processes, ASTM International, 2012.

[10] G. Bandini, M. Polidori, A. Gerschenfeld, D. Pialla, S. Li, W. Ma, P. Kudinov, M. Jeltsov, K. Kööp, K. Huber, et al., Assessment of systems codes and their coupling with cfd codes in thermal-hydraulic applications to innovative reactors, Nuclear Engineering and Design 281 (2015) 22-38.

[11] R. P. Martin, Relap5/mod3 code coupling model, Nuclear Safety 36 (2) (1995) 290-298.

[12] D. Aumiller, E. Tomlinson, R. Bauer, A coupled relap5-3d/cfd methodology with a proof-of-principle calculation, Nuclear Engineering and Design 205 (1-2) (2001) 83-90. 
[13] W. Li, X. Wu, D. Zhang, G. Su, W. Tian, S. Qiu, Preliminary study of coupling cfd code fluent and system code relap5, Annals of Nuclear Energy 73 (2014) 96-10\%.

[14] J. Herb, Coupling openfoam with thermo-hydraulic simulation code athlet, in: 9th OpenFOAM Workshop, Zagreb (Croatia), 2014.

[15] T. P. Grunloh, A. Manera, A novel domain overlapping strategy for the multiscale coupling of cfd with $1 d$ system codes with applications to transient flows, Annals of Nuclear Energy 90 (2016) 422-432.

[16] D. Martelli, N. Forgione, G. Barone, I. Di Piazza, Coupled simulations of the nacie facility using relap5 and ansys fluent codes, Annals of Nuclear Energy 101 (2017) 408-418.

[17] W. Weaver, E. Tomlinson, D. Aumiller, An executive program for use with relap5-3d(C), in: BT-3394, 2001 RELAP5 Users Seminar, Sun Valley, Idaho, 2001.

[18] D. Aumiller, E. Tomlinson, W. Weaver, An integrated relap5-3d and multiphase cfd code system utilizing a semi-implicit coupling technique, Nuclear engineering and design 216 (1-3) (2002) 77-87.

[19] D. Aumiller, F. Buschman, E. Tomlinson, D. Gill, Development of an integrated code system using r5exec and relap5-3d, Nuclear Technology 193 (1) (2016) 183-199.

[20] K. Zhang, V. H. Sanchez-Espinoza, The dynamic-implicit-additionalsource (dias) method for multi-scale coupling of thermal-hydraulic codes to enhance the prediction of mass and heat transfer in the nuclear reactor pressure vessel, International Journal of Heat and Mass Transfer 147 (2020) 118987.

[21] D. Ramajo, S. Corzo, N. Schiliuk, N. Nigro, 3d modeling of the primary circuit in the reactor pressure vessel of a phwr, Nuclear Engineering and Design 265 (2013) 356-365.

[22] S. Corzo, D. Ramajo, N. Nigro, 1/3d modeling of the core coolant circuit of a phwr nuclear power plant, Annals of Nuclear Energy 83 (2015) 368-39\%.

[23] D. Ramajo, S. Corzo, N. Nigro, A coupled model for two-phase simulation of a heavy water pressure vessel reactor, Int. J. of Mathematical, Computational, Physical, Electrical and Computer Engineering 9-11 (2015) 1-6.

[24] D. Pialla, D. Tenchine, S. Li, P. Gauthe, A. Vasile, R. Baviere, N. Tauveron, F. Perdu, L. Maas, F. Cocheme, et al., Overview of the 
system alone and system/cfd coupled calculations of the phenix natural circulation test within the thins project, Nuclear Engineering and Design 290 (2015) 78-86.

[25] C. Wang, H. Nilsson, J. Yang, O. Petit, 1d-3d coupling for hydraulic system transient simulations, Computer Physics Communications 210 (2017) 1-9.

[26] M. Aufiero, C. Fiorina, A. Laureau, P. Rubiolo, V. Valtavirta, Serpent-openfoam coupling in transient mode: simulation of a godiva prompt critical burst, Proceedings of $M E C+S N A+M C$ (2015) 19-23.

[27] G. F. Hewitt, G. L. Shires, T. R. Bott, Process heat transfer, Vol. 113, CRC press Boca Raton, FL, 1994.

[28] H. Jasak, A. Jemcov, Z. Tukovic, et al., Openfoam: A c++library for complex physics simulations, in: International workshop on coupled methods in numerical dynamics, Vol. 1000, IUC Dubrovnik, Croatia, 2007, pp. 1-20.

[29] S. F. Corzo, D. E. Ramajo, N. M. Nigro, High-rayleigh heat transfer flow: Thermal stratification analysis and assessment of boussinesq approach, International Journal of Numerical Methods for Heat $\&$ Fluid Flow 27 (9) (2017) 1928-1954.

[30] B. E. Launder, D. B. Spalding, The numerical computation of turbulent flows, Computer methods in applied mechanics and engineering 3 (2) (1974) 269-289.

[31] J. H. Ferziger, M. Perić, Computational methods for fluid dynamics, Vol. 3, Springer Berlin, 2002.

[32] W. Ambrosini, N. Forgione, J. Ferreri, M. Bucci, The effect of wall friction in single-phase natural circulation stability at the transition between laminar and turbulent flow, Annals of Nuclear Energy 31 (16) (2004) 1833-1865.

[33] F. Moukalled, L. Mangani, M. Darwish, et al., The finite volume method in computational fluid dynamics, Vol. 113, Springer, 2016.

[34] W. M. Kays, A. L. London, Compact heat exchangers.

[35] H. Gao, F. Gao, X. Zhao, J. Chen, X. Cao, Transient flow analysis in reactor coolant pump systems during flow coastdown period, Nuclear engineering and design 241 (2) (2011) 509-514.

[36] R. Uddin, Steady-state characteristics based model for centrifugal pump transient analysis, Annals of Nuclear Energy 21 (5) (1994) 321-324. 
[37] S. W. Churchill, H. H. Chu, Correlating equations for laminar and turbulent free convection from a horizontal cylinder, International journal of heat and mass transfer 18 (9) (1975) 1049-1053.

[38] A. Z. Mesquita, D. A. Palma, A. L. Costa, C. Pereira, M. A. Veloso, P. A. Reis, Experimental distribution of coolant in the ipr-r1 triga nuclear reactor core, International Nuclear Atlantic Conference - INAC 2011. 